\title{
Factores ambientales que influyen en el éxito de la inseminación artificial en la raza ovina Rasa Aragonesa
}

\author{
Tejedor, M.T. ${ }^{\circledR} ;$ Monteagudo, L.V.'; Laviña, A. ${ }^{2}$ y Macías, A. ${ }^{2}$
}

\begin{abstract}
'Departamento de Anatomía, Embriología y Genética Animal. Universidad de Zaragoza. Zaragoza. España.
${ }^{2}$ Asociación Nacional de Criadores de Ganado Ovino Selecto de Raza Rasa Aragonesa (ANGRA). Zuera. Zaragoza. España.
\end{abstract}

\section{RESUMEN}

\section{PALABRAS CLAVE ADICIONALES}

Manejo reproductivo.

Fecundidad.

Fertilidad.

Prolificidad.
El éxito de la inseminación artificial (IA) en ganado ovino se basa en los valores obtenidos de fecundidad, fertilidad, y prolificidad. El objetivo de este trabajo es valorar la importancia práctica sobre dicho éxito de diversos factores ambientales, ajenos al proceso de recogida y preparación del semen propiamente dicho: identidad del macho, ganadería, mes de la inseminación, temperatura ambiental, circunstancias ambientales, realización o no de flushing, manejo durante la inseminación, desarrollo de la inseminación, técnico inseminador, centro de recogida de semen, año, día tras el parto. Se han analizado datos de los últimos 10 años correspondientes a 5935 lotes, con un total de 58602 ovejas Rasa Aragonesa, procedentes de 172 explotaciones. Se han detectado numerosos factores con efectos significativos $(p<0,010)$ sobre el éxito de IA (macho, mes IA; temperatura ambiental, flushing, desarrollo de la IA y ganadería), pero, en general, cada uno de ellos explica por separado una pequeña parte de la variación en el éxito de la IA. Alcanzar los mejores resultados posibles en los protocolos de IA ovina requiere el control de dichos factores ambientales durante la inseminación; una buena formación y experiencia del inseminador y una total coordinación de la actuación de técnicos y ganaderos son imprescindibles.

\section{Ambient factors affecting artificial insemination success in Rasa Aragonesa sheep breed}

\section{SUMMARY}

Artificial insemination (Al) success in sheep is evaluated on the basis of obtained fecundity, fertility and prolificacy values. The aim of the present work is to estimate the practical influence of several ambient factors on Al success, other than those related to the actual collection and preparation of semen: inseminating ram, flock, month of $\mathrm{Al}$, ambient temperature, ambient conditions, flushing, management of $\mathrm{Al}$, development of $\mathrm{Al}$, technician, center of semen collection, year and lambing-Al interval. We analyzed data collected during the last 10 years from 58602 Rasa Aragonesa sheep, grouped in 5935 lots and belonging to 172 flocks. We detected significant effects $(p<0.010)$ on Al success for several factors: inseminating ram, ambient temperature, flushing, development of Al and farmt. However, each factor had only a little effect on $\mathrm{Al}$ success. To get the best possible results in $\mathrm{Al}$, all these factors must be controlled; good training and experience of technicians and famer-technician coordination are essential conditions.

\section{INFORMACIÓN}

Cronología del artículo.

Recibido/received: 12.04 .15

Aceptado/Accept: 09.03.16

Online: 15.09 .16

Correspondencia a los autores/Contact e-mail:

Hejedor@unizar.es

\section{INTRODUCCIÓN}

La Inseminación Artificial (IA) es una herramienta esencial en la mejora genética, tanto en la etapa de valoración de reproductores como en la difusión de la mejora a las diferentes ganaderías (Gourley y Riese, 1990). El éxito de la inseminación artificial en un rebaño se va- lora de acuerdo a tres conceptos diferentes aunque relacionados; fecundidad, fertilidad y prolificidad (Langford et al., 1979; Paulenz et al., 2002; Anel et al., 2003). La fecundidad es la proporción de corderos nacidos por oveja inseminada. No debe confundirse con la fertilidad, que es la proporción de hembras inseminadas que llegan al parto. Finalmente, la prolificidad es el pro- 
medio de corderos nacidos en cada parto. A lo largo de los últimos diez años, ANGRA (Asociación Nacional de Criadores de Ganado Ovino Selecto de Raza Rasa Aragonesa) ha recogido datos referentes a factores que afectan a la IA; el objetivo de este trabajo es valorar la influencia real de cada uno de ellos, es decir, su importancia práctica.

La IA en ovino no se encuentra tan generalizada como en otras especies (bovino, porcino), debido a sus irregulares resultados en cuanto a fertilidad y a las dificultades de uso de semen congelado (Anel et al., 2006). Por otra parte, David et al. (2008) encontraron valores muy bajos de heredabilidad para la fertilidad en ovino (entre 0,001 y 0,078), por lo que la mejora genética de los resultados de la IA a través de la selección poligénica clásica sería difícil. Consecuentemente, sería esperable conseguir una mejora de los resultados de la IA actuando sobre los factores no genéticos que podrían afectarle. Se ha hecho especial hincapié en la sincronización y detección de celos (Shelton, 1990; Abecia et al., 2011), así como en la calidad del semen (Santolaria et al., 2015). En este sentido, el objetivo de este trabajo es valorar la importancia práctica sobre dicho éxito de diversos factores ambientales, ajenos a los factores dependientes de la hembra y del macho indicados: identidad del macho, ganadería, mes de la inseminación, temperatura ambiental, circunstancias ambientales, realización o no deflushing, manejo durante la inseminación, desarrollo de la inseminación, técnico inseminador, centro de recogida de semen, año, día tras el parto.

\section{MATERIAL Y MÉTODOS}

Se han analizado 5935 lotes, que incluyen un total de 58602 ovejas, correspondientes a 172 explotaciones. En cada intervención se registró una amplia lista de factores que podrían influir en el éxito de la IA: identidad del macho usado en la IA, ganadería, mes de la inseminación, temperatura ambiental, circunstancias ambientales, realización o no de flushing, manejo durante la inseminación, desarrollo de la inseminación (correcto o incorrecto), técnico inseminador, centro de recogida de semen, año, día tras el parto. El paquete estadístico IBM $®$ SPSS $®$ versión 22 se ha utilizado para estimar la media, la desviación típica (DS) y las correlaciones bivariadas de fertilidad, fecundidad y prolificidad, así como para elaborar los diagramas de cajas de las variables consideradas. El procedimiento GLM (Modelo lineal general) se ha utilizado

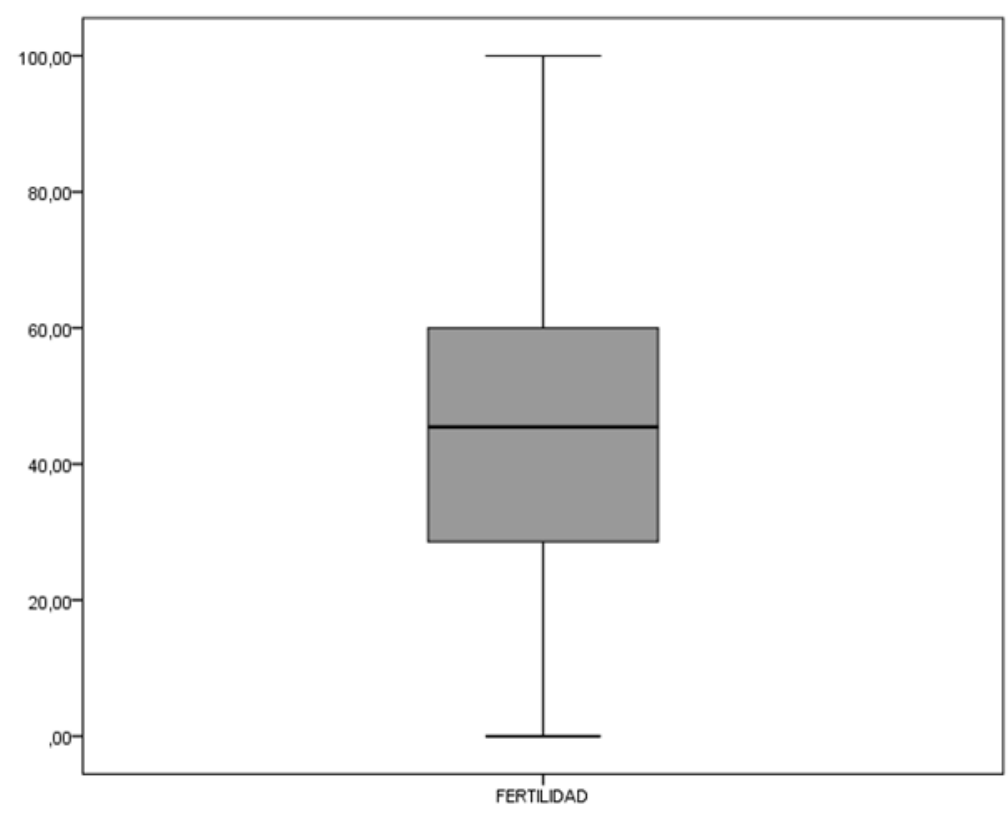

Figura 1. Diagrama de caja para fertilidad (Fertility box plot).

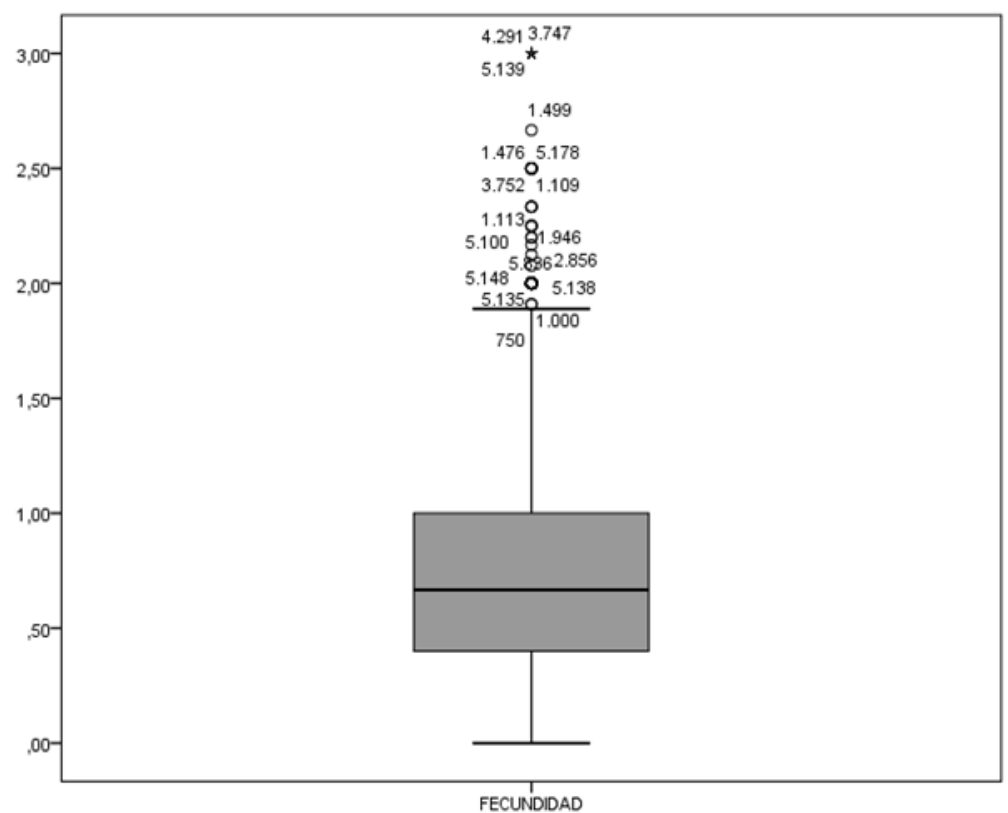

Figura 2. Diagrama de caja para fecundidad (Fecundity box plot).

para valorar la influencia de los factores considerados en el éxito de la IA; puede incorporar efectos aleatorios (macho y ganadería) y fijos (resto de factores considerados) así como interacciones. Valores de $p<0,050$ se consideraron como estadísticamente significativos. Cuando se detectaron efectos significativos de los factores, el tamaño del efecto se estimó mediante $\eta^{2}$ (porcentaje de la variabilidad total de la variable dependiente explicado por la variabilidad del factor) y mediante la diferencia 
de las medias entre los valores de los factores con su correspondiente intervalo de confianza al $95 \%$ (Petrie y Watson, 1999).

\section{RESULTADOS Y DISCUSIÓN}

Se han obtenido unos valores medios de fertilidad, fecundidad y prolificidad de de $44,9 \pm 23,5 \% ; 0,70 \pm 0,42$ corderos / oveja inseminada y $1,45 \pm 0,53$ corderos / parto, respectivamente (medias por lote $\pm \mathrm{DS}$ ). Las figuras 1, 2 y 3 muestran los diagramas de cajas de las tres variables consideradas. Mientras que en fertilidad la distribución de valores es simétrica y carece de outliers, tanto la fecundidad como la prolificidad muestran asimetría acusada y numerosos outliers, con máximos de 3 corderos / oveja inseminada y 3 corderos/parto, respectivamente.

En la tabla I se presentan los factores que han mostrado un efecto estadísticamente significativo $(\mathrm{p}<0,050)$ sobre fertilidad, fecundidad y prolificidad alcanzadas en los protocolos de IA y que son controlables durante el proceso, así como la estimación del tamaño del efecto. En el caso de las ganaderías y de los machos, no pudo estimarse la diferencia entre las medias ni su intervalo de confianza, al tratarse de factores aleatorios. En el caso de la fertilidad, no se ha detectado ninguna interacción significativa ( $p>0,05)$. Sin embargo, tanto en fecundidad como en prolificidad la interacción flushing * temperatura ambiental fue significativa $(p=0,011$ y $p=0,001$, respecti-

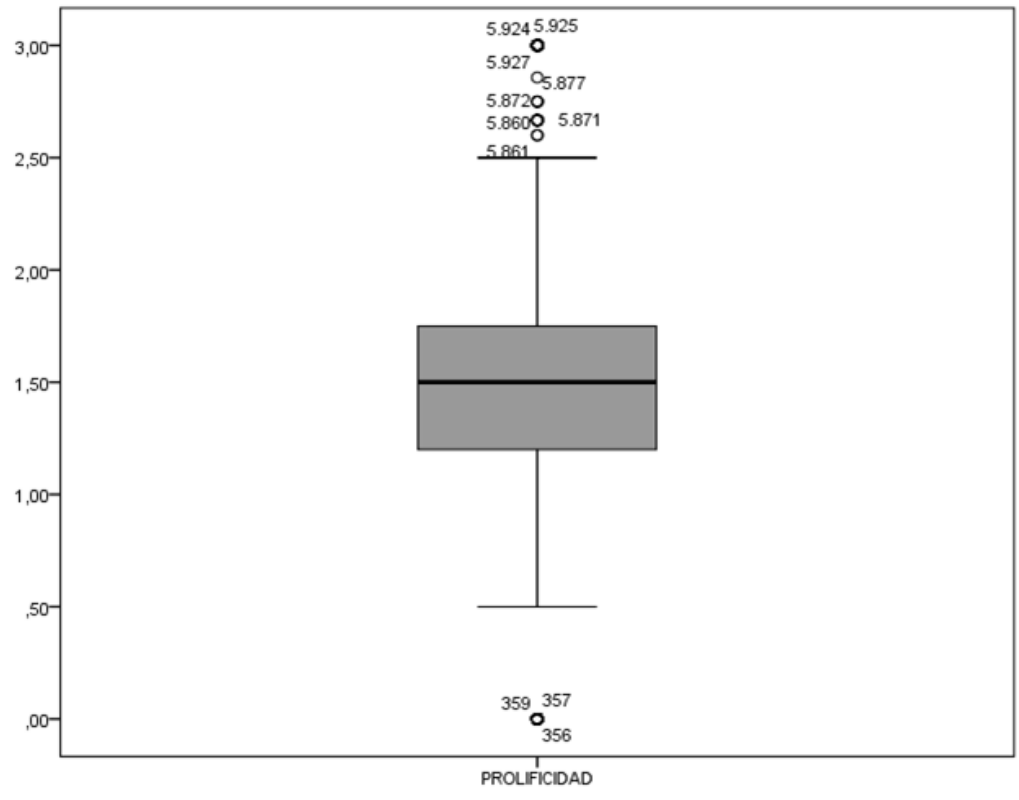

Figura 3. Diagrama de caja para prolificidad (Prolificacy box plot).

vamente). Tanto para la fecundidad como para la prolificidad, los mayores valores se alcanzan para la realización de flushing a una temperatura de entre $5^{\circ} \mathrm{C}$ y $25^{\circ} \mathrm{C}$. Estas interacciones significativas explicarían, la ausencia de efecto significativo del flushing sobre la fecundidad y del flushing y de la temperatura ambiental sobre la prolificidad.

Según los resultados obtenidos, una temperatura ambiental en el momento de la IA de $5{ }^{\circ} \mathrm{C}-25^{\circ} \mathrm{C}$ favorece el éxito de la IA. Estos resultados son similares a los obteni-

Tabla I. Factores ambientales con efecto estadísticamente significativo $(\mathrm{p}<0,010)$ sobre el éxito de IA (Ambient factors $(p<0.010)$ with significant effects on Al success).

\begin{tabular}{|c|c|c|c|c|c|c|c|c|c|}
\hline \multirow[t]{2}{*}{ Factor } & \multicolumn{3}{|c|}{ Fertilidad (\%) } & \multicolumn{3}{|c|}{$\begin{array}{c}\text { Fecundidad } \\
\text { (corderos/ oveja inseminada) }\end{array}$} & \multicolumn{3}{|c|}{ Prolificidad (corderos/ parto) } \\
\hline & $\eta^{2}(\%)$ & DM & IC95 & $\eta^{2}(\%)$ & $\mathrm{DM}$ & IC95 & $\eta^{2}(\%)$ & $\mathrm{DM}$ & IC95 \\
\hline $\begin{array}{l}\text { Temperatura ambiental } \\
5-25^{\circ} \mathrm{C} \text { vs otras }\end{array}$ & 0,2 & 2,36 & $0,88-3,84$ & 0,2 & 0,05 & $0,02-0,08$ & NS & - & - \\
\hline $\begin{array}{l}\text { Mes IA } \\
\text { Jul-Nov vs Dic-Jun }\end{array}$ & 0,6 & 4,29 & $2,87-5,70$ & 1,4 & 0,12 & $0,09-0,14$ & 0,7 & 0,11 & $0,08-0,15$ \\
\hline $\begin{array}{l}\text { Flushing } \\
\text { Si vs No }\end{array}$ & 0,2 & 2,70 & $1,18-4,21$ & NS & - & - & NS & - & - \\
\hline $\begin{array}{l}\text { Desarrollo de IA } \\
\text { Correcto vs incorrecto }\end{array}$ & 0,2 & 14,29 & $5,44-23,15$ & 0,2 & 0,25 & $0,09-0,41$ & NS & - & - \\
\hline Ganadería & 18,3 & - & - & 17,7 & - & - & 7,8 & - & - \\
\hline Macho & 4,7 & - & - & 4,5 & - & - & 3,7 & - & - \\
\hline
\end{tabular}

$\eta^{2}$ : Cada casilla muestra la magnitud del efecto (NS= $\left.p>0,05\right)$.

$\mathrm{DM}=$ Diferencia entre las medias; IC95= Intervalo de confianza al $95 \%$. 
Tabla II. Correlaciones bivariadas entre fertilidad, fecundidad y prolificidad (Bivariate correlations among fertility, fecundity and prolificacy).

\begin{tabular}{lcc}
\hline & Fecundidad & Prolificidad \\
\hline Fertilidad & $0,90^{* *}$ & $0,40^{* *}$ \\
Fecundidad & - & $0,63^{* *}$ \\
\hline${ }^{* *}<<0,01$. & & \\
\hline
\end{tabular}

dos por Santolaria et al. (2014) quienes detectaron un descenso de probabilidad de gestación por encima de los $30{ }^{\circ} \mathrm{C}$ en Rasa aragonesa. Palacios y Abecia (2015) detectaron también que los factores metorológicos afectaban a la fertilidad tras la IA, de forma que las temperaturas no extremas la favorecían. En otras especies de rumiantes se han obtenido resultados similares. Flamenbaum y Galon (2010) hallaron un efecto negativo de las altas temperaturas del verano (por encima de $30{ }^{\circ} \mathrm{C}$ ) sobre la fertilidad en vacas lecheras. En cabras, el efecto ambiental más importante relativo al éxito de la IA fue la época del año, con un efecto claramente negativo de las olas de calor (Furstoss et al., 2015). Abecia et al. (2016) encontraron que la temperatura y la lluvia tenían un efecto significativo sobre la fertilidad en cabras.

El éxito de la IA es mayor en los meses de Julio a Noviembre (tabla I). Estos resultados están de acuerdo con los de un estudio previo en Rasa aragonesa, en el que Palacín et al. (2012) observaron que la fertilidad disminuía en las inseminaciones de primavera (marzo-junio).

Se ha detectado un efecto significativo tanto de la granja como del macho en el éxito de la IA. Resultados similares fueron obtenidos por Anel et al. (2005); Arrebola et al. (2009); Palacín et al. (2012) y Santolaria et al. (2014).

Se detectan numerosos factores con efectos significativos sobre el éxito de IA, pero en su mayoría se trata de efectos de pequeña magnitud; en general, cada uno de ellos explica por separado una pequeña parte de la variación en el éxito de la IA. El balance coste/beneficio de realizar cambios sólo sería cuantificable en el caso del flushing. El flushing (incremento en el aporte energético tres semanas antes y tres después de la cubrición de las ovejas con un índice de condición corporal inferior a 3.0) favorece la ovulación y reduce las pérdidas embrionarias (Keisler y Buckrell, 1997). Para ello es necesario contar con alimento de buena calidad, granos de cereales, praderas verdes, heno, entre otros; en caso contrario, es indispensable considerar la suplementación con pienso específico. Hemos estimado el coste del flushing con maíz y alfalfa en 1,8-3,6€/oveja, siendo de $10 € /$ oveja si se realiza con pienso específico. El precio del ternasco de Aragón en 2015 fue de 72 €/canal. En caso de flushing, según nuestros resultados, se producirían 2,70 partos más por cada 100 ovejas y, considerando el valor medio de prolificidad (1,45 corderos/parto), se obtendrían, de promedio, 3,91 corderos más por cada 100 ovejas. A precio de mercado, esto supone un promedio de $282 €$ más por cada 100 ovejas; el balance coste/ beneficio sólo resultaría favorable en el caso de flushing más barato ( $180 € / 100$ ovejas), con un beneficio de $102 €$ por cada 100 ovejas.

Para tranquilidad de los ganaderos, hay que aclarar que fueron pocos los protocolos que se clasificaron como incorrectos, pero es de señalar que esta incorrección tendría un efecto significativo evitable sobre la fecundidad y la fertilidad, por lo que deberá prestarse especial atención a la preparación de todos los elementos relacionados con la explotación que van a intervenir en el manejo de los ejemplares durante la IA, de forma que se desarrolle de forma ordenada y tranquila.

En casi todos los casos, excepto para el mes de la inseminación, la magnitud de los efectos es mayor sobre la fecundidad y la fertilidad, es decir sobre el inicio de la gestación y sobre su mantenimiento hasta el parto, que sobre la prolificidad (número de corderos nacidos en cada parto). De hecho, la correlación es muy alta entre fecundidad y fertilidad (tabla II), por lo que los mismos factores afectan a ambas en el mismo sentido y puede afirmarse que las mejoras obtenidas en el índice de fertilidad se reflejan también en incrementos de la fecundidad. La menor correlación de ambas con la prolificidad (tabla II) explica por qué algunos factores no afectan a esta última. Así, el desarrollo de la inseminación no influye en el número de corderos nacidos en cada parto. Algo similar ocurriría con el flushing y la temperatura ambiental durante la inseminación; cada factor en particular no influye en el número de corderos nacidos en cada parto, pero su interacción, como se ha indicado más arriba, tiene una influencia significativa. Mejorar notablemente la fecundidad o la fertilidad de los protocolos exigirá atender a numerosas fuentes de variación, tanto por parte de las ganaderías como de los equipos técnicos que realizan el proceso de IA.

\section{CONCLUSIONES}

Ha de atenderse a todos los factores que afectan al correcto desarrollo de la IA, más eficaz entre Julio y Diciembre, evitando los momentos de más calor o frío ambiental y practicando el flushing si el estado general de las ovejas lo aconseja.

Las diferencias entre los resultados obtenidos por las diferentes explotaciones son también altamente significativas. Por este motivo, la comunicación entre técnicos y ganaderos es esencial: todos los detalles del manejo relacionados con la IA (incluido el periodo anterior y posterior a la misma) deben optimizarse para garantizar el éxito de la IA. 


\section{BIBLIOGRAFÍA}

Abecia, J.A.; Forcada, F. and González-Bulnes, A. 2011. Pharmaceutical control of reproduction in sheep and goats. Vet Clin North Am Food Anim Pract, 27: 67-79.

Abecia, J.A.; Arrebola, F.; Macías, A.; Laviña, A.; GonzálezCasquet, O.; Benítez, F. and Palacios, C. 2016. Temperature and rainfall are related to fertility rate after spring artificial insemination in small ruminants. Int J Biometeorol, 2016. Epub ahead of print DOI: 10.1007/s00484-016-1150-y.

Anel, L.; de Paz, P.; Álvarez, M.; Chamorro, C.A.; Boixo, J.C.; Manso, A.; González, M.; Kaabi, M. and Anel, E. 2003. Field and in vitro assay of three methods for freezing ram semen. Theriogenology, 60: 1293-1308.

Anel, L.; Kaabi, M.; Abroug, B.; Alvarez, M.; Anel, E.; Boixo, J.C.; de la Fuente, L.F. and de Paz, P. 2005. Factors influencing the success of vaginal and laparoscopic artificial insemination in Churra ewes: a field assay. Theriogenology, 63: 1235-1247.

Anel, L.; Alvarez, M.; Martinez-Pastor, F.; Garcia-Macias, V.; Anel, E. and de Paz, P. 2006. Improvement strategies in ovine artificial insemination. Reprod Domest Anim, 41 (Suppl 2): 30-42.

Arrebola, F.A.; Abecia, J.A.; Forcada, F.; Garcia, A.; Martín, R.A. and Mesa, O. 2009. Effects of annual rainfall and farm on lamb production after treatment with melatonin implants in Merino sheep: a 4-year study. N Z Vet J, 57: 141-145.

David I.; Robert-Granié,C.; Manfredi, E.; Lagriffoul, G. and Bodin, L. 2008. Environmental and genetic variation factors of artificial insemination success in French dairy sheep. Animal, 2: 979-986. Flamenbaum, I. and Galon, N.2010. Management of heat stress to improve fertility in dairy cows in Israel. J Reprod Dev, 56 (Suppl): S36-S41.

Furstoss, V.; David, I.; Fatet, A.; Boissard, K.; Clément, V. and Bodin, L. 2015. Genetic and non-genetic factors related to the success of artificial insemination in dairy goats. Animal, 9: 1935-1942.
Gourley, D.D. and Riese, R.L. 1990. Laparoscopic artificial insemination in sheep. Vet Clin North Am Food Anim Pract, 6: 615-633.

Keisler, D.H. and Buckrell, B.C. 1997. Breeding strategies. Chapter 88. In: Robert S. Youngquist (Ed.). Current therapy in large animal theriogenology. $1^{\text {st. }}$ edition. Elsevier. Amsterdam. The Nederland. pp. 649-661.

Langford, G.A.; Marcus, G.J.; Hackett, A.J.; Ainsworth, L.; Peters, H.F. and Wolynetz, M.S. 1979. A comparison of fresh and frozen semen in the insemination of confined sheep. Can J Anim Sci, 59: 685-691

Palacín, I.; Yániz, J.L.; Fantova, E.; Blasco, M.E.; Quintín-Casorrán, F.J.; Sevilla-Mur, E. and Santolaria P.2012. Factors affecting fertility after cervical insemination with cooled semen in meat sheep. Anim Reprod Sci, 132: 139-144.

Palacios, C. and Abecia, J.A. 2015. Meteorological variables affect fertility rate after intrauterine artificial insemination in sheep in a seasonal-dependent manner: a 7-year study. Int J Biometeorol, 59: 585-592.

Paulenz, H.; Adnøy, T.; Fossen, O.H.; Söderquist, L. and Berg, K.A. 2002. Effect of deposition site and sperm number on the fertility of sheep inseminated with liquid semen. Vet Rec, 150: 299-302.

Petrie, A. and Watson, P. 1999. Statistics for veterinary and animal science. Blackwell Science. Oxford, UK. 243 pp.

Santolaria, P.; Yániz, J.; Fantova, E.; Vicente-Fiel, S. and Palacín, I. 2014. Climate factors affecting fertility after cervical insemination during the first months of the breeding season in Rasa Aragonesa ewes. Int J Biometeorol, 58: 1651-1655.

Santolaria, P.; Vicente-Fiel, S.; Palacín, I.; Fantova, E.; Blasco, M.E.; Silvestre M.A. and Yániz, J.L. 2015. Predictive capacity of sperm quality parameters and sperm subpopulations on field fertility after artificial insemination in sheep. Anim Reprod Sci, 163: 82-88

Shelton, J.N. 1990. Reproductive technology in animal production. Rev Sci Tech, 9: 825-45. 